\title{
Mild solutions of non-Lipschitz stochastic integrodifferential evolution equations
}

\author{
Mamadou Abdoul Diop, Tomás Caraballo*and Aziz Mané
}

In this work we study the existence and uniqueness of mild solutions for stochastic partial integrodifferential equations under local non-Lipschitz conditions on the coefficients. Our analysis makes use of the theory of resolvent operators as developed by R. Grimmer [3] as well as a stopping time technique. Our results complement and improve several earlier related works. An example is provided to illustrate the theoretical results. Copyright (C) 2009 John Wiley \& Sons, Ltd.

Keywords: Resolvent operators, Co-semigroup, Local non-Lipschitz condition, Stochastic partial integrodifferential evolution equations, Mild solutions.

\section{Introduction}

In this work, we study the existence and uniqueness of mild solutions to the following partial stochastic integrodifferential equation:

$$
\left\{\begin{array}{l}
d X(t)=\left[A X(t)+\int_{0}^{t} B(t-s) X(s) d s+f(t, X(t))\right] d t+g(t, X(t)) d W(t) \\
X(0)=x_{0} \in \mathbb{H}
\end{array}\right.
$$

where $A: D(A) \subset \mathbb{H} \rightarrow \mathbb{H}$ is a closed linear operator, for all $t \geq 0, B(t)$ is a closed linear operator with domain $D(B(t)) \supset D(A)$. the mappings $f:[0,+\infty) \times \mathbb{H} \rightarrow \mathbb{H}, g:[0,+\infty) \times \mathbb{H} \rightarrow L_{2}^{0}(\mathbb{K}, \mathbb{H})$ are appropriate functions.

Stochastic evolution equations (SEEs) have attracted much attention over the last decades because of their practical applications in many areas such as physics, population dynamics, electrical engineering, medicine, biology, ecology and other areas of science and engineering. Especially, several researchers pay much attention to the existence and uniqueness to SEEs under some kind of weak sufficient conditions $[7,1]$. The so-called non-Lipschitz condition can be considered indeed as such a weaker sufficient condition and it also has a wider range of potential applications. This condition has been investigated in several papers. Yamada [11] and $\mathrm{Xu}$ [12] studied the solutions to stochastic differential equations (SDEs) under Yamada type nonLipschitz condition. Taniguchi[9] generalized the Yamada type non-Lipschitz condition and studied the existence and uniqueness of solutions to SDEs under Taniguchi type non-Lipschitz condition which includes the special result of [11]. Pardoux[7] has studied the energy solutions which satisfy the energy equality to the non-Lipschitz SEEs. Cao et al. [1] considered the existence and uniqueness of solutions to non-Lipschitz SEEs driven by Poisson jumps. Similar to study the case of local Lipshitz condition, the SEEs only with local non-Lipshitz condition proposed by Taniguchi[9] has recently attracted considerable attention. Taniguchi [10] discussed the existence and uniqueness of solutions to local non-Lipshitz SEEs driven by Wiener process. Ren [8] considered the mild solutions of neutral semilinear stochastic functional dynamical systems with local non-Lipschitz coefficients driven by Wiener process. Motivated by the previously mentioned works, our aim is to exploit the properties of the resolvent operators theory introduced in [3] jointly with a stopping time technique to prove the existence and uniqueness of mild solutions to Eq.(1). In this way, we can improve the results obtained in [2] for a neutral functional model which contains our model in the sense that we need to impose less assumptions to obtain the results (see Remark 3 for more details).

M. Abdoul Diop: Université Gaston Berger de Saint-Louis, UFR SAT, Département de Mathématiques, B.P234, Saint-Louis, Sénégal (Email: mamadouabdoul.diop@ugb.edu.sn)

T. Caraballo: Dpto. Ecuaciones Diferenciales y Análisis Numérico, Universidad de Sevilla, Apdo. de Correos 1160, 41080-Sevilla, Spain (Email: caraball@us.es) A. Mané: Université Gaston Berger de Saint-Louis, UFR SAT, Département de Mathématiques, B.P234, Saint-Louis, Sénégal (Email: azizmanesn@outlook.fr) * Correspondence to: Email: caraball@us.es. Postal Address: Dpto. Ecuaciones Diferenciales y Análisis Numérico, Universidad de Sevilla, Apdo. de Correos 1160, 41080-Sevilla, Spain. Tel: +34 954557998 
The outline of this work is as follows. In Section 2, we introduce the necessary notation and spaces, and review some basic definitions and results for partial integrodifferential equations. In Section 3, the existence and uniqueness of local mild solutions to our model are discussed and, finally, in Section 4, we provide an example to illustrate the basic results of this work.

\section{Preliminaries}

\subsection{Wiener process}

Let $\mathbb{H}$ be separable Hilbert space with the norm |.| and \|. $\|$, respectively. We denote by (.,.) the inner product in $\mathbb{H}$.

Let $\mathbb{K}$ be another separable Hilbert space with the inner product $(.,.) \mathbb{K}, L(\mathbb{K}, \mathbb{H})$ the space of all bounded linear operators from $\mathbb{K}$ to $\mathbb{H}$, and let $Q \in L(\mathbb{K}, \mathbb{K})$ be a nonnegative self-adjoint operator. Furthermore, let $L_{2}^{0}(\mathbb{K}, \mathbb{H})$ denote the space of all $\xi \in L(\mathbb{K}, \mathbb{H})$ such that $\xi \sqrt{Q}$ is a Hilbert-Schmidt operator and so $\operatorname{tr}\left(\xi Q \xi^{*}\right)<\infty$. The norm is given by

$$
\|\xi\|_{L_{2}^{0}}^{2}:=\|\xi \sqrt{Q}\|_{H S}^{2}=\operatorname{tr}\left(\xi Q \xi^{*}\right)
$$

Then $\xi$ is called a $Q$-Hilbert-Schmidt operator from $\mathbb{K}$ to $\mathbb{H}$. We note that if $Q=I$, then $L_{2}^{0}(\mathbb{K}, \mathbb{H})$ implies $L_{2}(\mathbb{K}, \mathbb{H})$.

Let $(\Omega, P, \mathcal{F})$ be a complete probability space on which an increasing and right continuous family $\left(\mathcal{F}_{t}\right)_{t \in[0, \infty]}$ of complete sub- $\sigma$-algebra of $\mathcal{F}$ is defined. $\mathcal{F}_{0}$ contains all the null subsets of $\mathcal{F}$. Let $\beta_{n}(t)(n=1,2,3, \ldots)$ be a sequence of real valued one-dimensional standard Brownian motions mutually independent on $(\Omega, P, \mathcal{F})$. When one considers the following series

$$
\sum_{n=1}^{\infty} \beta_{n}(t) e_{n}, t \geq 0
$$

where $\left\{e_{n}\right\}(n=1,2,3, \ldots)$ is a complete orthonormal basis in $\mathbb{K}$, this series does not necessarily converge in the space $\mathbb{K}$. Thus we consider a $\mathbb{K}$-valued stochastic process $W(t)$ given formally by the following series :

$$
W(t):=\sum_{n=1}^{\infty} \beta_{n}(t) \sqrt{Q} e_{n}, t \geq 0, Q \in L(\mathbb{K}, \mathbb{K}) .
$$

If $Q=$ I, we have to assume that there exists a Hilbert space $\mathbb{K}_{1} \supset \mathbb{K}$ such that it converges in $\mathbb{K}_{1}$, being the inclusion $\mathbb{K}_{1} \supset \mathbb{K}$ a Hilbert-Schmidt embedding. However in this paper, and from now, we suppose that operator $Q \in L(\mathbb{K}, \mathbb{K})$ is a nonnegative self-adjoint trace-class operator. Thus, the series converges in the space $\mathbb{K}$, that is, it holds that $W(t) \in L^{2}(\Omega ; \mathbb{K})$. Let $v \in \mathbb{K}$. The inner product $(W(t), v)_{\mathbb{K}}$ given by the above $\mathbb{K}$-valued stochastic process $W(t)$ satisfies the conditions of a cylindrical Wiener process since

$$
(W(t), v)_{\mathbb{K}}=\left(\sum_{n=1}^{\infty} \beta_{n}(t) \sqrt{Q} e_{n}, v\right)_{\mathbb{K}}, v \in \mathbb{K}, t \geq 0 .
$$

Therefore, when the operator $Q \in L(\mathbb{K}, \mathbb{K})$ is a nonnegative self-adjoint trace-class operator, we usually call the above $W(t)$ the $\mathbb{K}$-valued $Q$-cylindrical Wiener process with a covariance operator $Q$. Next, let $\phi(s)$ be an $L_{2}^{0}(\mathbb{K}, \mathbb{H})$ valued $\mathcal{F}_{t}$-adapted stochastic process with $\mathbb{E}\left[\int_{0}^{t}\|\phi(s)\|_{L_{2}^{0}}^{2} d s\right]<\infty$. Then we define the stochastic integral

$$
\int_{0}^{t} \phi(s) d W(s) \in \mathbb{H}, \quad t \geq 0
$$

of $\phi$ with respect to the $\mathbb{K}$-valued $Q$-cylindrical Wiener process $W(t)$ by

$$
\left(\int_{0}^{t} \phi(s) d W(s), v\right):=\sum_{n=1}^{\infty} \int_{0}^{t}\left(\phi(s) \sqrt{Q} e_{n}, v\right) d \beta_{n}(s)
$$

for any $v \in \mathbb{H}$ using the Ito integral with respect to $\beta_{n}(s)$.

\subsection{Partial integrodifferential equation in Banach space}

In the present section, we recall some definitions, notations and properties needed in the sequel.

In what follows, $\mathbb{H}$ will denote a Banach space, $A$ and $B(t)$ are closed linear operators on $\mathbb{H}$. $Y$ represents the Banach space $\mathcal{D}(A)$, the domain of operator $A$, equipped with the graph norm

$$
\|y\|_{Y}:=\|A y\|+\|y\| \text { for } \quad y \in Y .
$$


The notation $C([0,+\infty) ; Y)$ stands for the space of all continuous function from $[0,+\infty)$ into $Y$. We then consider the following Cauchy problem

$$
\left\{\begin{array}{l}
v^{\prime}(t)=A v(t)+\int_{0}^{t} B(t-s) v(s) d s \text { for } t \geq 0 \\
v(0)=v_{0} \in \mathbb{H}
\end{array}\right.
$$

Definition 1 ([3]) A resolvent operator of Eq. (2) is a bounded linear operator valued function $R(t) \in \mathcal{L}(\mathbb{H})$ for $t \geq 0$, satisfying the following properties:

1. $R(0)=I$ and $\|R(t)\| \leq N e^{\beta t}$ for some constant $N$ and $\beta$.

2. For each $x \in \mathbb{H}, R(t) x$ is strongly continuous for $t \geq 0$.

3. For $x \in Y, R(). x \in C^{1}([0,+\infty) ; \mathbb{H}) \cap C([0,+\infty) ; Y)$ and

$$
\begin{aligned}
R^{\prime}(t) x & =A R(t) x+\int_{0}^{t} B(t-s) x d s \\
& =R(t) A x+\int_{0}^{t} R(t-s) x d s \text { for } t \geq 0
\end{aligned}
$$

For additional details on resolvent operators, we refer the reader to [3, 4]. The resolvent operator plays an important role to study the existence of solutions and to establish a variation of constants formula for non-linear systems. For this reason, we need to know when the linear system (2) possesses a resolvent operator. Theorem 1 below provides a satisfactory answer to this problem.

Firstly, we impose the following assumptions on the operators $A$ and $B$ :

(H1) $A$ is the infinitesimal generator of a $C_{0}-\operatorname{semigroup~}(T(t))_{t \geq 0}$ on $\mathbb{H}$

(H2) For all $t \geq 0, \mathrm{~B}(\mathrm{t})$ is a continuous linear operator from $(Y,\|\| Y$.$) into \left(\mathbb{H},\|.\|_{\mathbb{H}}\right)$. Moreover, there exists an integrable function $c:[0,+\infty) \rightarrow \mathbb{R}^{+}$such that for any $y \in Y, t \mapsto B(t) y$ belongs to $W^{1,1}([0,+\infty), \mathbb{H})$ and

$$
\left\|\frac{d}{d t} B(t) y\right\|_{\mathbb{H}} \leq c(t)\|y\|_{Y} \text { for } y \in Y \text { and } t \geq 0 .
$$

We recall that $W^{k, p}(\Omega)=\left\{\tilde{\omega} \in L^{p}(\Omega): D^{\alpha} \tilde{\omega} \in L^{p}(\Omega), \forall|\alpha| \leq k\right\}$, where $D^{\alpha} \tilde{\omega}$ is the weak $\alpha$-th partial derivative of $\tilde{\omega}$.

Theorem 1 ([3]) Assume that hypotheses $(\mathbf{H 1})$ and $(\mathbf{H} 2)$ hold. Then Eq. (2) admits a resolvent operator $(R(t))_{t \geq 0 .}$

Theorem 2 ([5]) Assume that hypotheses (H1) and $\mathbf{( H 2 )}$ hold. Let $T(t)$ be a compact operator for $t>0$. Then, the corresponding resolvent operator $R(t)$ of $E q$. (2) is continuous for $t>0$ in the operator norm, namely, for $t_{0}>0$, it holds that $\lim _{h \rightarrow 0}\left\|R\left(t_{0}+h\right)-R\left(t_{0}\right)\right\|=0$.

In the sequel, we recall some results on the existence of solutions for the following integro-differential equation

$$
\left\{\begin{array}{l}
v^{\prime}(t)=A v(t)+\int_{0}^{t} B(t-s) v(s) d s+q(t) \text { for } \quad t \geq 0 \\
v(0)=v_{0} \in \mathbb{H}
\end{array}\right.
$$

where $q:[0,+\infty[\rightarrow \mathbb{H}$ is continuous function.

Definition 2 A continuous function $v:[0,+\infty) \rightarrow \mathbb{H}$ is said to be a strict solution of Eq. (3) if

1. $v \in C^{1}([0,+\infty) ; \mathbb{H}) \cap C([0,+\infty) ; Y)$,

2. $v$ satisfies Eq. (3) for $t \geq 0$.

Remark 1 From this definition we deduce that $v(t) \in \mathcal{D}(A)$, and the function $B(t-s) v(s)$ is integrable, for all $t>0$ and $s \in[0,+\infty)$.

Theorem 3 ([3]) Assume that hypotheses $\mathbf{( H 1 )}$ and $\mathbf{( H 2 )}$ hold. If $v$ is a strict solution of Eq. (3), then the following variation of constant formula holds

$$
v(t)=R(t) v_{0}+\int_{0}^{t} R(t-s) q(s) d s \text { for } t \geq 0 .
$$

Accordingly, we can establish the following definition. 
Definition 3 A function $v:[0,+\infty) \rightarrow \mathbb{H}$ is called mild solution of Eq. (3), for $v_{0} \in \mathbb{H}$, if $v$ satisfies the variation of constants formula (4).

The next theorem provides sufficient conditions ensuring the regularity of solutions of the Eq.(3).

Theorem 4 Let $q \in C^{1}([0,+\infty) ; \mathbb{H})$ and $v$ be defined by (4). If $v_{0} \in \mathcal{D}(A)$, then $v$ is a strict solution of Eq. (3).

\section{Existence and uniqueness of mild solution}

In this section, we prove that there is a unique local mild solution for Eq. (1) under a minimal set of assumptions. We begin by introducing the following concept of mild solution.

Definition 4 A process $\{X(t), t \in[0, T]\}, 0 \leq T<+\infty$, is called a mild solution of (1) if :

(i) $X(t)$ is $\mathcal{F}_{t}$-adapted, $t \geq 0$ with $\int_{0}^{T}|X(u)|_{\mathbb{H}}^{2} d u<\infty$ a.s ;

(ii) $X(t) \in \mathbb{H}$ has continuous paths on $t \in[0, T]$ a.s., and, for each $t \in[0, T], X(t)$ satisfies the integral equation

$$
X(t)=R(t) x_{0}+\int_{0}^{t} R(t-s) f(s, X(s)) d s+\int_{0}^{t} R(t-s) g(s, X(s)) d W(s)
$$

for any $X(0)=x_{0} \in \mathbb{H}$.

Now we can list the following assumptions, some of them were used in the paper [2] where a stochastic neutral functional integrodifferential equation with delay is analyzed. It is worth emphasizing that the equation in [2] contains our model (1), as a particular case, by simply making the delay equal zero as well as the neutral term:

(H3) (1) (the growth condition) There exists a function $H(t, r): \mathbb{R}^{+} \times \mathbb{R}^{+} \rightarrow \mathbb{R}^{+}$such that $H(t, r)$ is locally integrable in $t \geq 0$ for any fixed $r \geq 0$ and is continuous, monotone nondecreasing and concave in $r$ for any fixed $t \in[0, T]$.

Furthemore, for any fixed $t \in[0, T]$ and $u \in \mathbb{H}$, the following inequality is satisfied:

$$
|f(t, u)|^{2}+|g(t, u)|_{L_{2}^{0}}^{2} \leq H\left(t,|u|^{2}\right), t \in[0, T],
$$

(2) for any constant $\gamma>0$, the differential equation

$$
\frac{d \theta}{d t}=\gamma H(t, \theta), t \in[0, T]
$$

has a solution $\theta(t)=\theta\left(t ; 0, \theta_{0}\right)$ on $[0, T]$ for any initial value $\theta_{0}$.

(H4) (1) (the local condition) For any integer $N>0$ there exists a function $G_{N}: \mathbb{R}^{+} \times \mathbb{R}^{+} \rightarrow \mathbb{R}^{+}$such that $G_{N}(t, r)$ is locally integrable in $t \in[0, T]$ for any fixed $r \geq 0$ and is continuous, monotone nondecreasing and concave in $r$ for any fixed $t \in[0, T]$ with $G_{N}(t, 0)=0$, Futhermore, the following inequality is satisfied: for any $u, v \in \mathbb{H}$ with $|u|,|v| \leq N$,

$$
|f(t, u)-f(t, v)|^{2}+|g(t, u)-g(t, v)|_{L_{2}^{0}}^{2} \leq G_{N}\left(t,|u-v|^{2}\right), t \in[0, T]
$$

(2) For any constant $\gamma>0$, if a nonnegative function $z(t)$ satisfies that

$$
z(t) \leq \gamma \int_{0}^{t} G_{N}(s, z(s)) d s
$$

for all $t \in[0, T]$, then $z(t) \equiv 0$ holds for any $t \in[0, T]$.

(H5) (1) (the global condition) There exists a function $G(t, r): \mathbb{R}^{+} \times \mathbb{R}^{+} \rightarrow \mathbb{R}^{+}$such that $G(t, r)$ is locally integrable in $t \in[0, T]$ for any fixed $r \geq 0$ and is continuous, monotone nondecreasing and concave in $r$ for any fixed $t \in[0, T]$. Furthermore, the following inequality is satisfied: for any $u, v \in \mathbb{H}$,

$$
|f(t, u)-f(t, v)|^{2}+|g(t, u)-g(t, v)|_{L_{2}^{0}}^{2} \leq G\left(t,|u-v|^{2}\right), t \in[0, T],
$$

(2) for any constant $\gamma>0$, if a nonnegative function $z(t)$ satisfies that

$$
z(t) \leq \gamma \int_{0}^{t} G(s, z(s)) d s
$$

for all $t \in[0, T]$, then $z(t) \equiv 0$ holds for any $t \in[0, T]$.

Remark 2 Observe that the first condition in $\mathbf{( H 4 )}$ is a generalization of the following Lipschitz condition: 
(1) (the locally Lipschitz condition) for any fixed integer $N>0$, there exists an $L_{N}>0$ such that for any $u, v \in \mathbb{H}$ with $|u| \leq N$ and $|v| \leq N$,

$$
|f(t, u)-f(t, v)|^{2}+|g(t, u)-g(t, v)|_{L_{2}^{0}}^{2} \leq L_{N}|u-v|^{2}, t \in[0, T] .
$$

In what follows, $c>0$ will denote a suitable positive real number which may be different from line to line and even in the same line.

Remark 3 As we have already mentioned, our model is a particular case of the equation analyzed by Diop et al. [2]. For this reason, using the technique in [2] (namely a Picard iteration method) we can prove in a straightforward way the following theorem for (1), under the assumptions of global non-Lipschitz and growth conditions on the coefficients $f$ and $g$.

Theorem 5 (see [2]) Assume that (H1), (H2), (H3) and $\mathbf{( H 5 )}$ are satisfied. Then there exists a unique mild solution solution $X(t)$ to $(1)$.

However, using a different technique, we will be able to improve the result provided by Theorem 5. Indeed, we will prove that the existence and uniqueness of mild solution to (1) holds true by assuming (H4) instead of $\mathbf{( H 3 )}$ and (H5). The proof is based in the theory of resolvent semigroups as well as a stopping time technique. We are therefore on position to prove the main theorem in this paper.

Theorem 6 Assume that $(\mathbf{H 1}),(\mathbf{H} 2)$ and $\mathbf{( H 4 )}$ are satisfied. Then there exists a unique mild solution $X(t)$ to $(1)$.

Proof. Let $N$ be a natural integer and let $T_{0} \in(0, T)$. We define the sequence of functions $\left\{f_{N}(t, u)\right\}$ and $\left\{g_{N}(t, u)\right\}$ for $(t, u) \in\left[0, T_{0}\right] \times \mathbb{H}$ as follows:

$$
\begin{gathered}
f_{N}(t, u)=\left\{\begin{array}{cc}
f(t, u) & \text { if }|u| \leq N, \\
f\left(t, \frac{N u}{|u|}\right) & \text { if } u>N,
\end{array}\right. \\
g_{N}(t, u)=\left\{\begin{array}{cc}
g(t, u) & \text { if }|u| \leq N, \\
g\left(t, \frac{N u}{|u|}\right) & \text { if } u>N,
\end{array}\right.
\end{gathered}
$$

then, the functions $\left\{f_{N}\right\}$ and $\left\{g_{N}\right\}$ satisfy $(\mathbf{H} 3)$ and the following inequality

$$
\left|f_{N}(t, u)-f_{N}(t, v)\right|^{2}+\left|g_{N}(t, u)-g_{N}(t, v)\right|_{L_{2}^{0}}^{2} \leq G_{N}\left(t,|u-v|^{2}\right), u, v \in \mathbb{H}, t \in\left[0, T_{0}\right]
$$

Thus, by Theorem 5, there exist the unique mild solutions $X_{N}(t)$ and $X_{N}(t)$, respectively to the following stochastic evolution equations:

$$
\begin{gathered}
X_{N}(t)=R(t) x_{0}+\int_{0}^{t} R(t-s) f_{N}\left(s, X_{N}(s)\right) d s+\int_{0}^{t} g_{N}\left(s, X_{N}(s)\right) d W(s) \\
X_{N+1}(t)=R(t) x_{0}+\int_{0}^{t} R(t-s) f_{N+1}\left(s, X_{N+1}(s)\right) d s+\int_{0}^{t} g_{N+1}\left(s, X_{N+1}(s)\right) d W(s)
\end{gathered}
$$

Define the stopping times

$$
\begin{aligned}
& \sigma_{N}:=T_{0} \wedge \inf \left\{t \in[0, T]:\left|X_{N}(t)\right| \geq N\right\} \\
& \sigma_{N+1}:=T_{0} \wedge \inf \left\{t \in[0, T]:\left|X_{N+1}(t)\right| \geq N+1\right\}, \\
& \tau_{N}:=\sigma_{N} \wedge \sigma_{N+1} . \\
& X_{N+1}(t)-X_{N}(t)=\quad \int_{0}^{t} R(t-s) f_{N+1}\left(s, X_{N+1}(s)\right) d s-\int_{0}^{t} R(t-s) f_{N}\left(s, X_{N}(s)\right) d s \\
& \quad+\int_{0}^{t} R(t-s) g_{N+1}\left(s, X_{N+1}(s)\right) d W(s)-\int_{0}^{t} R(t-s) g_{N}\left(s, X_{N}(s)\right) d W(s) \\
&=\quad \int_{0}^{t} R(t-s)\left[f_{N+1}\left(s, X_{N+1}(s)\right)-f_{N}\left(s, X_{N}(s)\right)\right] d s \\
&+\int_{0}^{t} R(t-s)\left[g_{N+1}\left(s, X_{N+1}(s)\right)-g_{N}\left(s, X_{N}(s)\right)\right] d W(s) .
\end{aligned}
$$

Recall that, for $a, b \in \mathbb{H},|a+b|^{2} \leq 2\left(|a|^{2}+|b|^{2}\right)$. Hence,

$$
\begin{aligned}
\mathbb{E} \sup _{0 \leq s \leq t \wedge \tau_{N}}\left|X_{N+1}(s)-X_{N}(s)\right|^{2}= & \mathbb{E} \sup _{0 \leq s \leq t \wedge \tau_{N}} \mid \int_{0}^{s} R(s-r)\left[f_{N+1}\left(r, X_{N+1}(r)\right)-f_{N}\left(r, X_{N}(r)\right)\right] d r \\
& +\left.\int_{0}^{s} R(s-r)\left[g_{N+1}\left(r, X_{N+1}(r)\right)-g_{N}\left(r, X_{N}(r)\right)\right] d W(r)\right|^{2}
\end{aligned}
$$




$$
\begin{aligned}
\mathbb{E} \sup _{0 \leq s \leq t \wedge \tau_{N}}\left|X_{N+1}(s)-X_{N}(s)\right|^{2} \leq & 2 \mathbb{E} \sup _{0 \leq s \leq t \wedge \tau_{N}}\left|\int_{0}^{s} R(s-r)\left[f_{N+1}\left(r, X_{N+1}(r)\right)-f_{N}\left(r, X_{N}(r)\right)\right] d r\right|_{\mathbb{H}}^{2} \\
& +2 \mathbb{E} \sup _{0 \leq s \leq t \wedge \tau_{N}}\left|\int_{0}^{s} R(s-r)\left[g_{N+1}\left(s, X_{N+1}(s)\right)-g_{N}\left(r, X_{N}(r)\right)\right] d W(r)\right|^{2} \\
\leq & I_{1}+I_{2} .
\end{aligned}
$$

For $0 \leq s \leq \tau_{N}$, we see that $f_{N+1}\left(s, X_{N}(s)\right)=f_{N}\left(s, x_{N}(s)\right)=f\left(s, x_{N}(s)\right)$, and $g_{N+1}\left(s, X_{N}(s)\right)=g_{N}\left(s, x_{N}(s)\right)=g\left(s, x_{N}(s)\right)$ Thus, by (H4) and the Jensen inequality, we obtain

$$
\begin{aligned}
I_{1} & =2 \mathbb{E} \sup _{0 \leq s \leq t \wedge \tau_{N}}\left|\int_{0}^{s} R(s-r)\left[f_{N+1}\left(r, X_{N+1}(r)\right)-f_{N}\left(r, X_{N}(r)\right)\right] d r\right|^{2} \\
& \leq M \mathbb{E} \sup _{0 \leq s \leq t \wedge \tau_{N}} \int_{0}^{s}\left|f_{N+1}\left(r, X_{N+1}(r)\right)-f_{N}\left(r, X_{N}(r)\right)\right|^{2} d r \\
& \left.\leq M \mathbb{E} \int_{0}^{t \wedge \tau_{N}} \sup _{0 \leq u \leq r} G_{N+1}\left(r, \mid X_{N+1}(u)\right)-\left.X_{N}(u)\right|^{2}\right) d r \\
& \left.\leq M \int_{0}^{t \wedge \tau_{N}} G_{N+1}\left(r, \mathbb{E}\left(\sup _{0 \leq u \leq r} \mid X_{N+1}(u)\right)-\left.X_{N}(u)\right|^{2}\right)\right) d r
\end{aligned}
$$

By (H4), Liu [[6], Theorem 1.2.6, page 14] and the Jensen inequality, there exists a positive constant $M_{1}$ such that

$$
\begin{aligned}
I_{2} & =2 \mathbb{E} \sup _{0 \leq s \leq t \wedge \tau_{N}}\left|\int_{0}^{s} R(s-r)\left[g_{N+1}\left(r, X_{N+1}(r)\right)-g_{N}\left(r, X_{N}(r)\right)\right] d W(r)\right|^{2} \\
& \left.\leq M_{1} \int_{0}^{t \wedge \tau_{N}} G_{N+1}\left(r, \mathbb{E}\left(\sup _{0 \leq u \leq r} \mid X_{N+1}(u)\right)-\left.X_{N}(u)\right|^{2}\right)\right) d r
\end{aligned}
$$

From (10)-(11), we have

$$
\left.\mathbb{E} \sup _{0 \leq s \leq t}\left|X_{N+1}\left(s \wedge \tau_{N}\right)-X_{N}\left(s \wedge \tau_{N}\right)\right|_{\mathbb{H}}^{2} \leq\left(M+M_{1}\right) \int_{0}^{t} G_{N+1}\left(r, \mathbb{E}\left(\sup _{0 \leq u \leq r} \mid X_{N+1}\left(u \wedge \tau_{N}\right)\right)-\left.x_{N}\left(u \wedge \tau_{N}\right)\right|_{\mathbb{H}} ^{2}\right)\right) d r
$$

By (H4-2), we have

$$
\mathbb{E}\left(\sup _{0 \leq s \leq t \wedge \tau_{N}}\left|X_{N+1}(s)-X_{N}(s)\right|^{2}\right)=0
$$

which means thats for $0 \leq t \leq T_{0} \wedge \tau_{N}$, we obtain

$$
X_{N+1}(t)=X_{N}(t) \quad \text { a.e. }
$$

From Chebyschev's inequality and the fact that $\mathbb{E}\left(\sup _{0 \leq t \leq T_{0}}\left\|X_{N}(t)\right\|^{2}\right) \leq K_{1}$, it follows that

$$
\begin{aligned}
P\left(\tau_{N} \leq T_{0}\right) & \left.\leq P\left(\sup _{0 \leq t \leq T_{0}}\left\|X_{N}(t)\right\|^{2}\right)>N\right) \\
& \leq \frac{\mathbb{E}\left(\sup _{0 \leq t \leq T_{0}}\left\|X_{N}(t)\right\|^{2}\right)}{N^{2}} \\
& \leq \frac{K_{1}}{N^{2}} \rightarrow 0 \text { as } N \rightarrow+\infty .
\end{aligned}
$$

Note that this states slightly more than convergence in probability of $\tau_{N} \rightarrow T_{0}$. One can find, for almost every $\omega \in \Omega$, an $N_{0}(\omega)$ such that $0<T_{0}<\tau_{N_{0}}$.

Define $X(t)$ by $X(t)=X_{N_{0}}(t), t \in[0, T]$. Since $X\left(t \wedge \tau_{N}\right)=X_{N}\left(t \wedge \tau_{N}\right)$, then we have

$$
\begin{aligned}
X\left(t \wedge \tau_{N}\right) & =R(t) x_{0}+\int_{0}^{t \wedge \tau_{N}} R(t-s) f_{N}(s, X(s)) d s+\int_{0}^{t \wedge \tau_{N}} R(t-s) g_{N}(s, X(s)) d W(s) \\
& =R(t) x_{0}+\int_{0}^{t \wedge \tau_{N}} R(t-s) f(s, X(s)) d s+\int_{0}^{t \wedge \tau_{N}} R(t-s) g(s, X(s)) d W(s) .
\end{aligned}
$$

Letting $N \rightarrow \infty$,

$$
X(t)=R(t) x_{0}+\int_{0}^{t} R(t-s) f(s, X(s)) d s+\int_{0}^{t} R(t-s) g(s, X(s)) d W(s) .
$$

Hence we have that $X(t)$ is a mild solution to (1). The proof is complete. 


\section{Example}

In this section, we provide an example to illustrate our main results. We consider the following stochastic partial functional integrodifferential equation:

$$
\left\{\begin{array}{l}
\frac{\partial}{\partial t} \beta(t, \xi)=\frac{\partial^{2}}{\partial \xi^{2}} \beta(t, \xi)+\int_{0}^{t} b(t-s) \frac{\partial^{2}}{\partial \xi^{2}} \beta(s, \xi) d s \\
\quad+\lambda(t) f_{1}(\beta(t, \xi)) \beta(t, \xi) d t+\gamma_{1} \beta(t, \xi) d W(t) \\
\beta(t, 0)=\beta(t, \pi)=0, \quad t \geq 0, \\
\beta(0, \xi)=\beta_{0}(\xi), \quad \xi \in[0, \pi],
\end{array}\right.
$$

where $W(t)$ denotes a $\mathbb{R}$-valued Brownian motion, $\lambda^{2}(t)>0$ is a locally integrable function, $f_{1}: \mathbb{R}^{+} \rightarrow \mathbb{R}$ is a bounded continuous functions, $b \in C^{1}\left(\mathbb{R}^{+}, \mathbb{R}\right)$ and $\beta_{0}:[0, \pi] \rightarrow \mathbb{R}$ is a given function such that $\beta_{0}(\cdot) \in L^{2}(0, \pi)$, is $\mathcal{F}_{0}$-measurable and satisfies $E\left\|\beta_{0}\right\|^{2}<\infty$.

Let $\mathbb{H}=L^{2}(0, \pi)$ with the norm $\|\cdot\|$ and $e_{n}:=\sqrt{\frac{2}{\pi}} \sin (n x), \quad(n=1,2,3, \cdots)$ denote a complete orthonormal basis in $\mathbb{H}$. We assume that there exists the product $u v \in \mathbb{H}$ for $u, v \in \mathbb{H}$. We note that there exists an $M \geq 1$ such that $|u v| \leq M\|u\|\|v\|$. Let $W(t):=\sum_{n=1}^{\infty} \sqrt{\lambda_{n}} \delta_{n}(t) e_{n}\left(\lambda_{n}>0\right)$, where $\delta_{n}(t)$ are one dimensional standard Brownian motion mutually independent of an usual complete probability space $\left(\Omega, \mathcal{F},\left\{\mathcal{F}_{t}\right\}_{t \geq 0}, \mathbb{P}\right)$.

Define $A: D(A) \subset \mathbb{H} \rightarrow \mathbb{H}$ by $A=\frac{\partial^{2}}{\partial z^{2}}$, with domain $D(A)=H^{2}(0, \pi) \cap H_{0}^{1}(0, \pi)$. Then

$$
A \hat{h}=-\sum_{n=1}^{\infty} n^{2}<\hat{h}, e_{n}>e_{n}, \hat{h} \in D(A),
$$

where $e_{n}, n=1,2,3, \cdots$, is also the orthonormal set of eigenvectors of $A$. It is well-known that $A$ is the infinitesimal generator of a strongly continuous semigroup $T(t)$ on $\mathbb{H}$, given by

$$
T(t) \hat{h}=\sum_{n=1}^{\infty} e^{-n^{2} t}<\hat{h}, e_{n}>e_{n} \text { for } \hat{h} \in \mathbb{H},
$$

which is compact.

Let $B: D(A) \subset \mathbb{H} \rightarrow \mathbb{H}$ be the operator defined by

$$
B(t)(\tilde{z})=b(t) A \tilde{z} \text { for } t \geq 0 \text { and } \tilde{z} \in D(A) \text {. }
$$

Let $\phi$ be as follows :

$$
\phi(x)=\left\{\begin{array}{l}
0, x=0 \\
c x\left(\log \frac{1}{x}\right), 0<x \leq \delta \\
c \delta\left(\log \frac{1}{\delta}\right), x>\delta
\end{array}\right.
$$

or

$$
\phi(x)=\left\{\begin{array}{l}
0, x=0, \\
c x\left(\log \frac{1}{x}\right)^{\frac{1}{3}} \log \log \frac{1}{x}, 0<x \leq \delta \\
c \delta\left(\log \frac{1}{\delta}\right)^{\frac{1}{3}} \log \log \frac{1}{\delta}, x>\delta
\end{array}\right.
$$

with $c>0$ and $0<\delta<1$ is sufficiently small. Assume that the following inequality is satisfied :

$$
\left|f_{1}(u)-f_{1}(v)\right|^{2} \leq \phi\left(|u-v|^{2}\right), u, v \in \mathbb{R}
$$

For $t \geq 0, \quad \xi \in[0, \pi]$ and $\psi$ a $\mathbb{H}$-valued function, define the operators $f, g: \mathbb{R}^{+} \times \mathbb{H} \rightarrow \mathbb{H}$ by

$$
\begin{aligned}
f(t, \psi)(\xi) & =\lambda(t) f_{1}(\psi)(\xi) \psi(\xi) \\
g(t, \psi)(\xi) & =\gamma_{1} \psi(\xi) .
\end{aligned}
$$

If we put

$$
\left\{\begin{array}{l}
X(t)=\beta(t, \xi) \text { for } t \geq 0 \text { and } \xi \in[0, \pi] \\
X(0)=x_{0},
\end{array}\right.
$$

then equation (13) takes the following abstract form

$$
\left\{\begin{array}{l}
d X(t)=\left[A X(t)+\int_{0}^{t} B(t-s) X(s) d s\right] d t+f(t, X(t)) d t+g(t, x(t)) d W(t) \\
X(0)=x_{0} \in \mathbb{H} .
\end{array}\right.
$$


Since $f_{1}(u)$ is a bounded function, there exists $B_{1}>0$ such that $|f(v)| \leq B_{1}$ for any $v \in H$. For any nonnegative real number $r \in \mathbb{R}^{+}$we set

$$
\begin{aligned}
\Phi_{N}(r): & =2 M^{2} N^{2} \phi(r), \\
\Psi(r): & =\left(2 M^{2} B_{1}^{2}+\gamma_{1}^{2} \operatorname{trace}(Q)\right) r .
\end{aligned}
$$

We define $\rho(t)$ by

$$
\rho(t):=\left\{\begin{array}{l}
1 \text { if } \lambda(t) \leq 1 \\
\lambda^{2}(t) \text { if } \lambda(t)>1
\end{array}\right.
$$

Then $\rho(t)$ is a locally integrable function. And we have that

$$
\left|\lambda(t) f_{1}(u) u-\lambda(t) f_{1}(v) v\right|^{2}+\left|\gamma_{1} u-\gamma_{1} v\right|_{L_{2}^{0}}^{2} \leq \rho(t)\left(\Phi_{N}\left(|u-v|^{2}\right)+\Psi\left(|u-v|^{2}\right)\right), u, v \in H .
$$

On the other hand, set

$$
a:=\left(2 M^{2} B_{1}^{2}+\gamma_{1}^{2} \operatorname{trace}(Q)\right) .
$$

Since $\phi$ is a concave function, it follows that $\phi(r) \geq \phi(1) r$ for $0 \leq r<1$. Thus it holds that

$$
\begin{aligned}
\int_{0^{+}}^{+\infty} \frac{1}{\Phi_{N}(r)+\Psi(r)} d r & =\int_{0^{+}}^{+\infty} \frac{1}{2 M^{2} N^{2} \phi(r)+a r} d r \\
& \geq \frac{\phi(1)}{\left(a+2 M^{2} N^{2} \phi(1)\right)} \int_{0^{+}}^{+\infty} \frac{1}{\phi(r)} d r \\
& =\infty
\end{aligned}
$$

Thus by the example of Lemma 3 [[9], p. 157] we have that $(\mathbf{H 4 )}$ holds. Therefore by Theorem 6 the proof of the example is complete.

\section{Acknowledgments}

We would like to thank the referees for their helpful suggestions which allowed us to improve the presentation of our paper.

This work has been partially supported by the Spanish Ministerio de Economía y Competitividad project MTM2011-22411, and the Consejería de Innovación, Ciencia y Empresa (Junta de Andalucía) under grant 2010/FQM314 and Proyecto de Excelencia P12-FQM-1492. The first author was partially supported by grant from Simons Foundation.

\section{References}

1. G. Cao, K. He, X. Zhang, Successive approximations of infinite dimensional SDES with jumps, Stoch. Dyn. 5(2005), 609-619.

2. M.A. Diop, K. Ezzinbi, M. Lo, Existence and uniqueness of mild solutions for some neutral stochastic partial functional integrodifferential equations with non-Lipschitz coefficients, Int. J. Math. Math. Sci. 2012, 748590 (2012). doi:10.1155/2012/748590

3. R. C. Grimmer, Resolvent operators for integral equations in a Banach space, Transactions of the American Mathematical Society, vol. 273, no. 1(1982),333-349.

4. R. C. Grimmer and A. J. Pritchard, Analytic resolvent operators for integral equations in Banach space, Journal of Differential Equations, vol. 50, no. 2(1983),234-259.

5. J. Liang, J.H. Liu, and T.J. Xiao, Nonlocal problems for integrodifferential equations, Continuous, Discrete and Impulsive Systems, Series A, Mathematical analysis, 15(2008), 815-824

6. K. Liu, Stability of Infinite Dimensional Stochastic Differential Equations with Applications, vol. 135, Chapman Hall; CRC, London, UK, 2006.

7. E. Pardoux, Stochastic partial differential equations and filtering of diffusion processes, Stochastics 3(1979)127-167.

8. Y. Ren, D. Sun, Second-order neutral stochastic evolution equations with infinite delay under Carathéodory conditions, J. Optim. Theory. Appl,147(2010),569-582.

9. T. Taniguchi, Successive approximations to solutions of stochastic differential equations, J. Differential Equations 96 (1992), $152-169$.

10. T. Taniguchi, The existence and uniqueness of energy solutions to local non-Lipschitz stochastic evolution equations, Journal of Mathematical Analysis and Applications, 360(2009),245-253.

11. T. Yamada,On the successive approximation of solutions of stochastic differential equations. J. Math. Kyoto Univ. 21(1981),501-515.

12. Y. Xu, B. Pei, G. Guo, Existence and stability of solutions to non-Lipschitz stochastic differential equations driven by Lévy noise, Applied Mathematics and Computation, 263(2015),398-409. 